\title{
Cerebral Hyperperfusion Syndrome Associated With Non-convulsive Status Epilepticus Following Superficial Temporal Artery-Middle Cerebral Artery Anastomosis
}

\author{
-Case Report-
}

\author{
Takeshi HAMAMURA, Takato MORIOKA, Tetsuro SAYAMA, Nobutaka MUKAE, \\ Shuji ARAKAWA*, Hironobu MAEDA**, and Tomio SASAKI*** \\ Departments of Neurosurgery, *Cerebrovascular Disease, and \\ **Radiology, Kyushu Rosai Hospital, Kitakyushu, Fukuoka; \\ ***Department of Neurosurgery, Graduate School of Medical Sciences, \\ Kyushu University, Fukuoka
}

\begin{abstract}
A 77-year-old man developed cerebral hyperperfusion syndrome with temporal deterioration of consciousness and worsening of left hemiparesis on the $6^{\text {th }}$ postoperative day following superficial temporal artery-middle cerebral artery (STA-MCA) anastomosis for right $M_{1}$ occlusion. Electroencephalography (EEG) revealed frequent ictal discharges in the right hemisphere, although convulsive seizures were not apparent. Administration of anticonvulsants was performed based on the diagnosis of non-convulsive status epilepticus (NCSE). Complete recovery from hyperperfusion syndrome was achieved with rapid improvement of EEG findings. The present case demonstrates the pathophysiological mechanism of hyperperfusion syndrome associated with NCSE after STA-MCA anastomosis.
\end{abstract}

Key words: cerebral hyperperfusion syndrome, non-convulsive status epilepticus, superficial temporal artery-middle cerebral artery anastomosis, ictal hyperperfusion, electroencephalography

\section{Introduction}

Carotid reconstruction surgery including carotid endarterectomy (CEA) or carotid stenting (CAS) can cause a rapid increase in cerebral blood flow $(\mathrm{CBF})$ in the chronic ischemic brain of patients with atherosclerotic diseases, resulting in complications such as cerebral hyperperfusion syndrome. ${ }^{3,15,19,22)}$ Patients with poor cerebrovascular reactivity are known to have higher risk for hyperperfusion syndrome. ${ }^{12,18,23)}$ The severity of intraoperative ischemia is also reported to be one of the predictive factors for hyperperfusion syndrome after CEA. ${ }^{12)}$ In contrast, hyperperfusion syndrome after superficial temporal arterymiddle cerebral artery (STA-MCA) anastomosis for atherosclerotic diseases is thought to be rare, since STAMCA anastomosis usually provides low-flow revascularization. ${ }^{23)}$ However, recent evidence suggests that STAMCA anastomosis for moyamoya disease as well as atherosclerotic disease can also result in symptomatic hyperperfusion. ${ }^{4,5,7,10,11,13)}$ However, the exact mechanism of hyperperfusion syndrome following STA-MCA anastomosis is still controversial. ${ }^{7,10)}$

We report a case of hyperperfusion syndrome associ-

Received March 3, 2010;

Accepted May 19, 2010 ated with non-convulsive status epilepticus (NCSE) following STA-MCA anastomosis for $\mathrm{M}_{1}$ occlusion, which demonstrates the exact temporal relationship between clinical findings, CBF measurements, and electroencephalography (EEG) findings in hyperperfusion syndrome.

\section{Case Report}

A 77-year-old right-handed man had been treated with 100 mg of aspirin under a diagnosis of multiple cerebral infarctions since age 75 years. He presented with left hemiparesis due to infarction in the right corona radiata 6 months previously. His hemiparesis gradually improved over several months of rehabilitation and he was introduced to our hospital to evaluate the capacity of cerebral perfusion reserve.

On admission, he had mild left hemiparesis, but was independent in activities of daily living. Fluid-attenuated inversion recovery (FLAIR) magnetic resonance (MR) imaging demonstrated a small infarction in the right corona radiata as well as periventricular hyperintensity on both sides (Fig. 1A). MR angiography and computed tomography (CT) angiography revealed occlusion (or severe stenosis) of the right $\mathrm{M}_{1}$ portion (Fig. 1B). All MR im- 

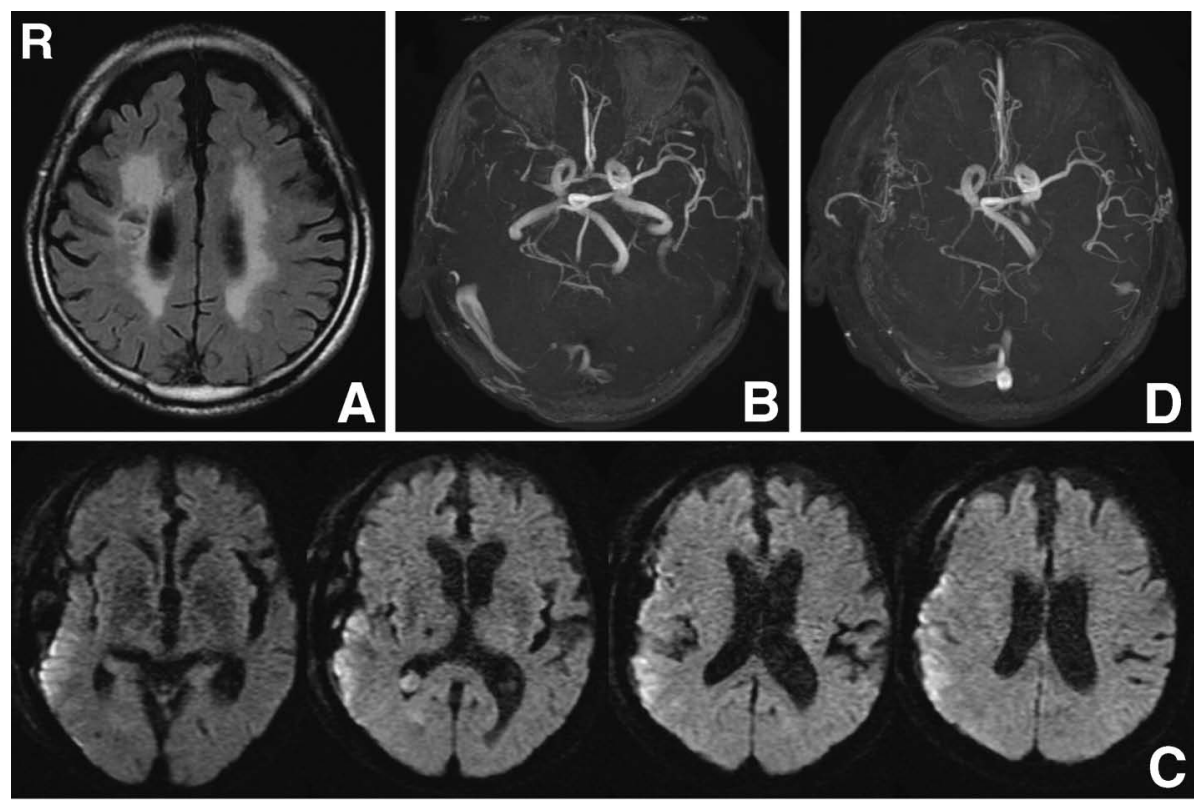

Fig. 1 A: Preoperative fluid-attenuated inversion recovery magnetic resonance (MR) image demonstrating a small infarction in the right corona radiata with arteriosclerotic change in the bilateral corona radiata. B: Preoperative MR angiogram indicating occlusion (or severe stenosis) of the right $M_{1}$ portion. C: Diffusion-weighted MR images on the $6^{\text {th }}$ postoperative day demonstrating gyriform cortical hyperintensity in the posterior part of the middle cerebral artery (MCA) territory. D: MR angiogram on the $6^{\text {th }}$ postoperative day showing patency of the superficial temporal artery-MCA anastomosis and hypervascularity of the peripheral branches of the right MCA.
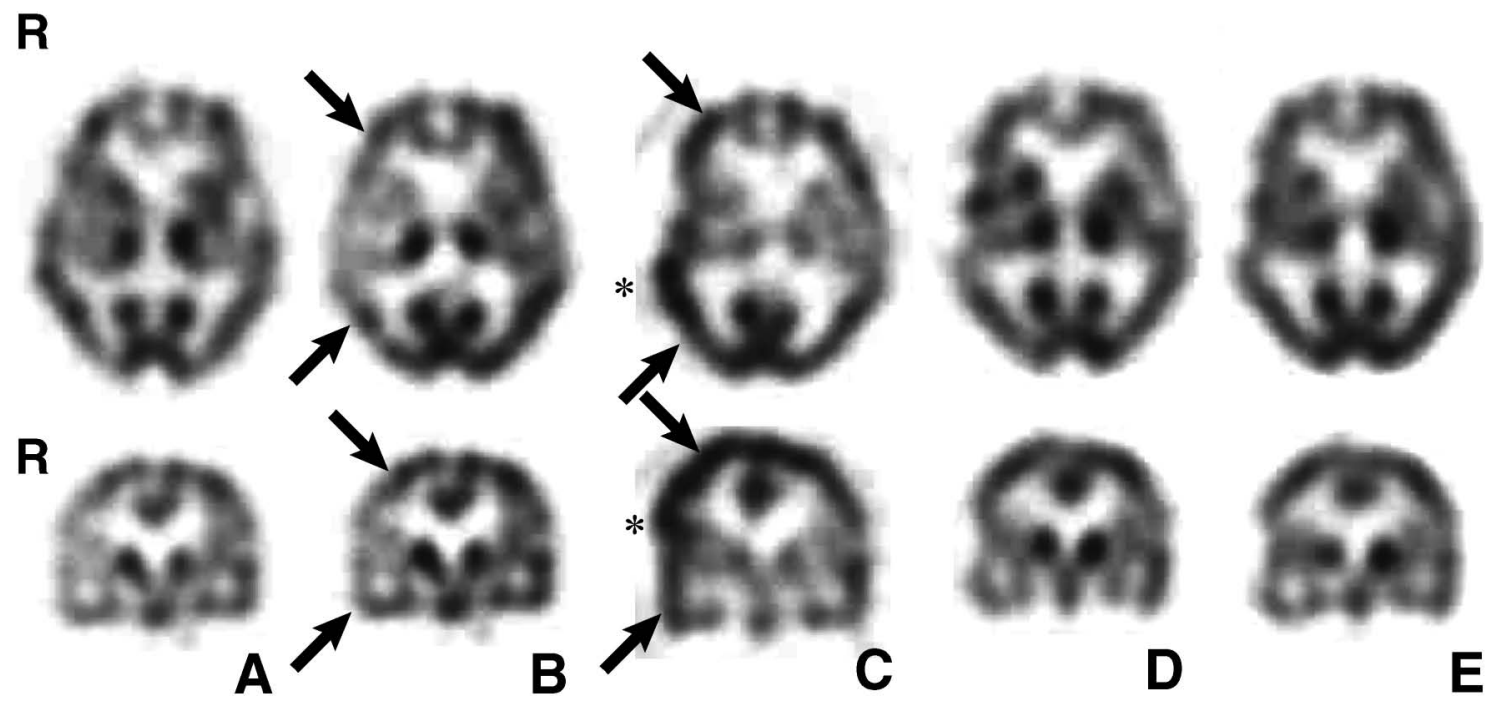

D

E

Fig. 2 A, B: Preoperative N-isopropyl-p-[123 I]iodoamphetamine single-photon emission computed tomography (IMP-SPECT) images at rest (A) and after acetazolamide loading (B) showing cerebral blood flow (CBF) in the right middle cerebral artery (MCA) territory is slightly decreased (A) and the vascular reserve capacity in the right MCA territory is markedly compromised (B, arrows). C: [99m Tc]-ethyl-cysteinate dimmer SPECT images on the $6^{\text {th }}$ postoperative day showing increased CBF in the MCA territory (arrows), especially in the posterior part of the MCA territory (asterisks). D, E: IMP-SPECT images on the $20^{\text {th }}$ postoperative day showing disappearance of the hyperperfusion of the right MCA territory (D), and improved vascular reserve capacity in the right MCA territory after acetazolamide loading (E). Upper and lower rows are axial and coronal views, respectively.

aging was performed with a $1.5 \mathrm{~T}$ MR unit (Signa Exite HD, version 12 software; GE Medical Systems, Milwaukee, Wis, U.S.A.). N-isopropyl-p-[123I]iodoamphetamine single-photon emission computed tomography (IMPSPECT) showed that vascular reserve capacity was markedly compromised in the right MCA territory (Fig. 2A, 


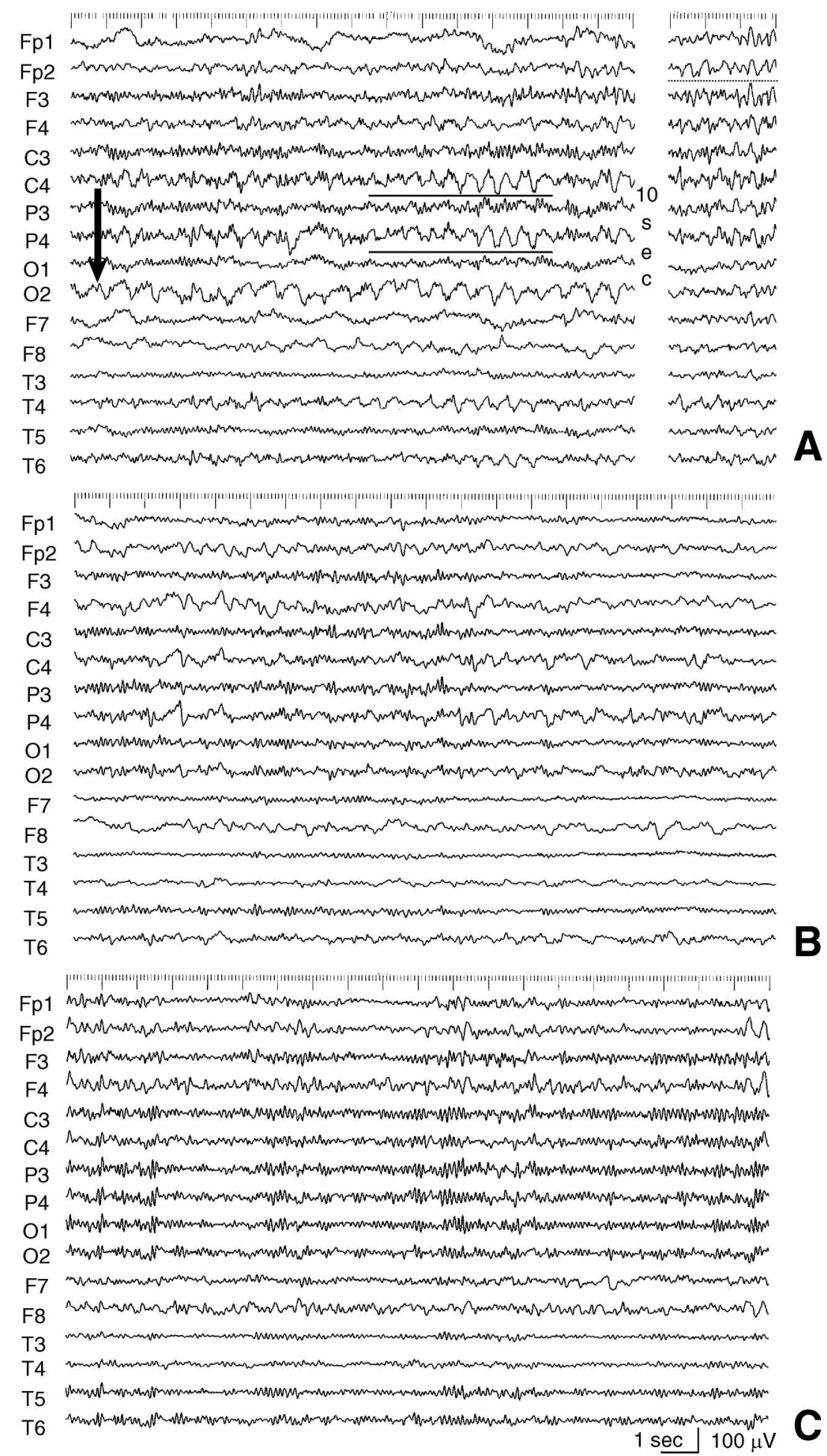

Fig. 3 A: Electroencephalograms (EEGs) on the $6^{\text {th }}$ postoperative day revealing frequent ictal discharges (rhythmic slow waves), which originate from the right occipital region (O2 of the International 10-20 system, arrow), extending to the right temporo-parietal region (P4 and C4, black lines) and then to the right frontal region (Fp2, dotted line). B: EEGs on the $9^{\text {th }}$ postoperative day showing no ictal discharge, although non-rhythmic slow waves were intermittently observed in the right fronto-parietal region. Relatively well-organized background activity was noted on O2, which is the ictal onset zone, on the $6^{\text {th }}$ postoperative day. C: EEGs on the $20^{\text {th }}$ postoperative day showing well-organized background activity in the bilateral occipital region, although intermittent slow waves were noted in the right frontal region (reference; ipsilateral earlobe). 

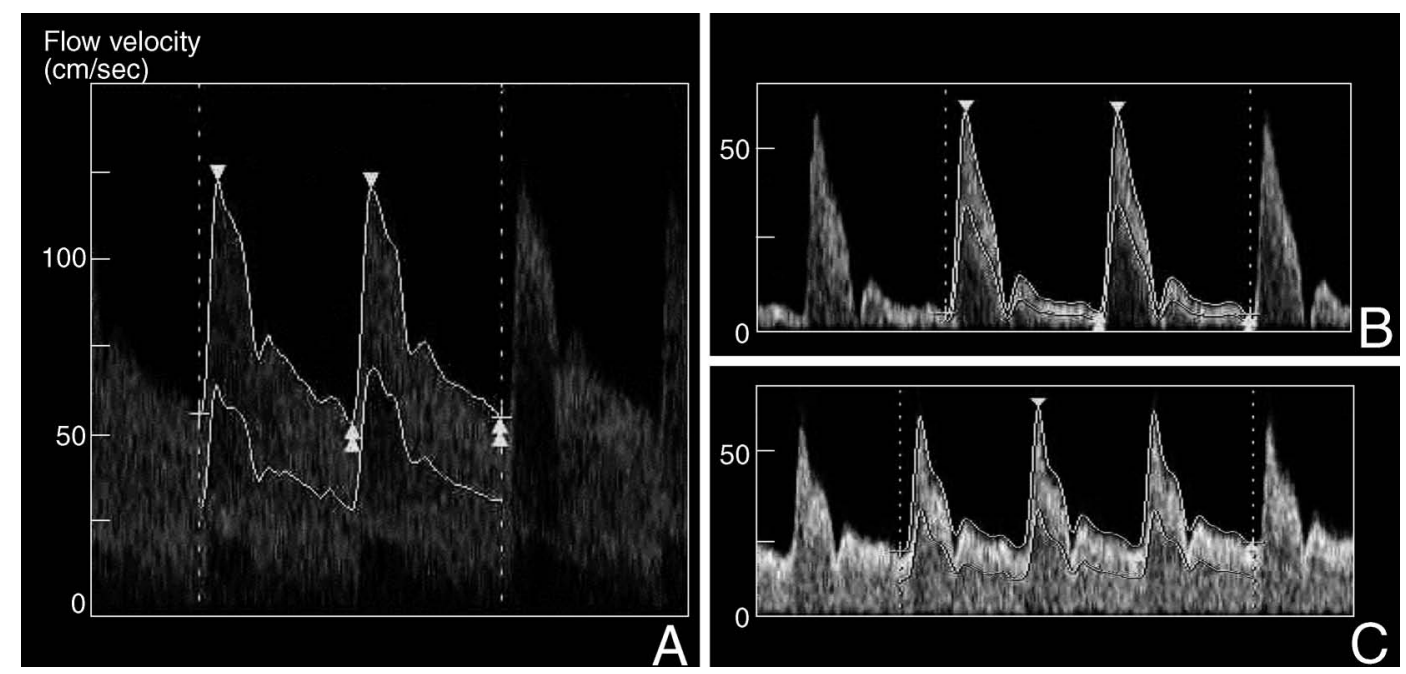

Fig. 4 A, B: Ultrasonograms of the right superficial temporal artery (STA) on the $8^{\text {th }}$ postoperative day (A) revealing marked increase in flow velocity in the systolic and end-diastolic periods $(120.2$ and $54.6 \mathrm{~cm} / \mathrm{sec}$, respectively) compared with the left $\mathrm{STA}$ (B; 57.6 and $4.9 \mathrm{~cm} / \mathrm{sec}$, respectively). C: Ultrasonograms of the right $\mathrm{STA}$ on the $32^{\text {nd }}$ postoperative day showing decrease in flow velocity in the systolic and end-diastolic periods $(62.2$ and $22.9 \mathrm{~cm} / \mathrm{sec}$, respectively).

B). CBF in the right and left MCA territories was 31.81 and $33.29 \mathrm{ml} / 100 \mathrm{~g} / \mathrm{min}$, respectively (Fig. 2A). Regional cerebrovascular reactivity (rCVR) after acetazolamide loading in the right and left MCA territories was -21.69 and $+11.79 \%$, respectively (Fig. $2 \mathrm{~B}$ ). Therefore, right STA-MCA anastomosis was scheduled.

Right fronto-temporo-parietal craniotomy was performed, and the parietal and frontal branches of the STA were anastomosed with the $\mathrm{M}_{4}$ portions on the frontal and temporal lobes, respectively. Occlusion times during the STA-MCA double anastomosis were 25 and 14 minutes, respectively. No cerebral contusion or subarachnoid hemorrhage was observed at craniotomy. The postoperative course was uneventful and systemic blood pressure was well controlled. However, the patient experienced deterioration of consciousness with confusion and worsening of left hemiparesis on the $6^{\text {th }}$ postoperative day.

$\mathrm{T}_{1}$ - or $\mathrm{T}_{2}$-weighted and FLAIR MR imaging failed to reveal additional abnormalities that could explain his neurological deterioration, but diffusion-weighted imaging demonstrated gyriform cortical hyperintensity in the posterior part of the MCA territory (Fig. 1C). The low apparent diffusion coefficients score of the right posterior temporal lobe was decreased by $33 \%$ compared with that in the contralateral side. MR angiography showed an apparently patent STA-MCA bypass as a thick hyperintense area compared with the preoperative images (Fig. 1D), as well as dilated branches of the MCA supplied by the bypass. [ $\left.{ }^{99 \mathrm{~m}} \mathrm{Tc}\right]$-ethyl-cysteinate dimmer (ECD)-SPECT observed an increase in CBF in the MCA territory, especially in the posterior part of the MCA territory (Fig. 2C).

EEG showed frequent ictal discharges on the right hemisphere, although convulsive seizures were not apparent. Ictal EEG patterns were stereotyped; rhythmic slow waves, which originated from the right occipital region (O2 of the International 10-20 system), extended to the right temporo-parietal region $(\mathrm{P} 4, \mathrm{C} 4)$ and then to the right frontal region (Fp2) (Fig. 3A). Duration of the seizure activities was 19-63 seconds (42.1 sec average). Fourteen episodes of ictal activities were recorded during 15 minutes. No paroxysmal activities were noted during the interictal period.

Ultrasonography examination of the STA demonstrated marked increases in flow velocities of the right STA in the systolic and end-diastolic periods $(120.2$ and $54.6 \mathrm{~cm} / \mathrm{sec}$, respectively) compared with the left STA (57.6 and 4.9 $\mathrm{cm} / \mathrm{sec}$, respectively) (Fig. 4A, B). Based on the diagnosis of hyperperfusion syndrome associated with NCSE, phenytoin was intravenously administered and a free radical scavenger (edaravone) was given.

The patient became well-orientated on the $9^{\text {th }}$ postoperative day. EEG detected no ictal discharge, although nonrhythmic slow waves were observed in the right frontoparietal region. Relatively well-organized background activities were noted in $\mathrm{O} 2$, the location of the ictal onset zone on the $6^{\text {th }}$ postoperative day (Fig. 3B). The patient's neurological condition had completely returned to that of the preoperative period on the $18^{\text {th }}$ postoperative day. EEG showed well-organized background activity in the bilateral occipital regions (O1 and $\mathrm{O} 2$ ), although intermittent slow waves were noted in the right frontal region (Fig. 3C). Diffusion-weighted imaging demonstrated disappearance of cortical hyperintensity in the MCA territory on the postoperative $20^{\text {th }}$ day. MR angiography showed that hypervascularity of the peripheral branches of the right MCA had disappeared. IMP-SPECT revealed disappearance of the hyperperfusion of the right MCA territory on the $20^{\text {th }}$ postoperative day (Fig. 2D). CBF was 55.73 and $55.57 \mathrm{ml} / 100 \mathrm{~g} / \mathrm{min}$ in the right and left MCA territories, respectively (Fig. 2A), and the vascular reserve capacity after acetazolamide loading in the right MCA territory had improved compared with that in the preoperative period 
(Fig. 2E). rCVR was +55.73 and $+55.57 \%$ in the right and left MCA territories, respectively. Ultrasonography of the right STA revealed decreased flow velocity of the right STA in the systolic and end-diastolic periods (62.2 and $22.9 \mathrm{~cm} / \mathrm{sec}$, respectively) on the $32^{\text {nd }}$ postoperative day (Fig. 4C). The patient was transferred to another hospital for further rehabilitation on the $40^{\text {th }}$ postoperative day.

\section{Discussion}

Seizures or epilepsy are one of the major symptoms in patients with hyperperfusion syndrome, ${ }^{17,22)}$ but surprisingly few reports describe the EEG findings. EEG obtained during hyperperfusion syndrome revealed periodic lateralizing epileptiform discharges (PLEDs) on the side of the brain ipsilateral to the CEA, even in the absence of seizures or during the postictal state. ${ }^{19)}$ NCSE is defined as a change in behavior and/or mental process from the baseline associated with ongoing seizure activity or continuous epileptiform discharges on EEG in the absence of convulsive symptoms. ${ }^{2,16,20)}$ Epileptic activity causes increased metabolic demand in the involved cortex, which is accompanied by temporarily increased regional cerebral perfusion. ${ }^{6,8)}$ Since PLEDs are one of the EEG patterns in patients with NCSE, ,29) the pathophysiology of "ictal" hyperperfusion could be similar to that observed in our case.

In the present case, EEG demonstrated frequent ictal discharges that originated from the right occipital region or temporo-occipital junction and extended to the central and parietal region. During NCSE, diffusion-weighted imaging demonstrated marked gyriform cortical hyperintensity of the fronto-temporo-parietal lobe. Furthermore, this hyperintensity did not completely match the vascular distribution of the MCA but almost coincided with the ictal onset and spreading zone. This cortical hyperintensity is one of the typical findings of partial status epilepticus and is thought to represent cytotoxic and vasogenic edema of the cortical lamina. ${ }^{14,21)}$ During NCSE, the hyperperfusion area on ECD-SPECT matched the vascular distribution of MCA, but the most prominent area was not the anastomosis site but the posterior temporal lobe, which corresponded to the findings of EEG and diffusion-weighted imaging.

Diagnosis of hyperperfusion syndrome is usually based on CBF findings obtained with SPECT or perfusion CT. ${ }^{3,5,10,15,22)}$ In this case, ECD-SPECT on the postoperative $6^{\text {th }}$ day clearly showed increased CBF, especially in the posterior part of the MCA territory, which disappeared on IMP-SPECT on the $20^{\text {th }}$ postoperative day. However, the procedures of SPECT and perfusion CT are expensive and not available at the bedside. Instead, measurement of flow velocity of $\mathrm{M}_{1}$ with transcranial ultrasonography is useful for evaluating the hyperperfusion state after CEA or CAS, ${ }^{22)}$ since ultrasonography is noninvasive and easily repeatable. Similarly, in our case, measurement of flow velocity of the STA with ultrasonography showed the hemodynamic state of the MCA territory through the STAMCA anastomosis as previously described.1)

Mortality is reported to be mainly dependent on underlying etiology and age, but NCSE carries a poor prognosis so prompt diagnosis and subsequent treatment is important. ${ }^{2,16,20)}$ SPECT and ultrasonography can clearly demonstrate the hyperperfusion state, but cannot detect neuronal function, especially neuronal excitability. In contrast to hyperperfusion after CEA, hyperperfusion is delayed after STA-MCA anastomosis. ${ }^{10)}$ A possible explanation is that the lower-flow bypass may take time to mature and so could affect the dysautoregulated brain. ${ }^{10)}$ Another explanation is the delayed occurrence of seizure activity, as in our case, although whether seizures or hyperperfusion are the first phenomenon cannot be determined.

After the diagnosis of hyperperfusion syndrome is made based on measurement of CBF, EEG is recommended even in the absence of seizures. With the diagnosis of status epilepticus, the first recommended treatment is rapid administration of antiepileptic drugs such as diazepam, phenobarbital, and phenytoin. ${ }^{16,20)}$ If prolonged seizure activity is noted, anesthesia with barbiturate or propofol may be indicated. ${ }^{16,20)}$ If increased CBF cannot overcome the increased metabolic demand during status epilepticus, neuronal ischemia can occur and free radicals are produced. Edaravone inhibits lipid peroxidation and vascular endothelial cell injury, and ameliorates brain edema and tissue injury. Antioxidants are beneficial in the treatment of acute stroke. Recently, pretreatment with edaravone decreased the incidence of post CEA hyperperfusion as measured by SPECT. ${ }^{18)}$

The present case suggests that ictal hyperperfusion should be excluded by EEG, even in the absence of seizures, after the diagnosis of hyperperfusion syndrome following STA-MCA anastomosis based on CBF measurement.

\section{Acknowledgments}

We thank Mses. Kuniko Yamamoto and Tomomi Kido, in the Department of Clinical Laboratory, and Mr. Kohji Miyazoe, in the Department of Radiology, Kyushu Rosai Hospital for supporting our study.

\section{References}

1) Arakawa S, Kamouchi M, Okada $Y$, Kishikawa K, Omae T, Inoue $\mathrm{T}$, Ibayashi $\mathrm{S}$, Fujishima $\mathrm{M}$ : Ultrasonographically predicting the extent of collateral flow through superficial temporal artery-to-middle cerebral artery anastomosis. AJNR Am J Neuroradiol 24: 886-891, 2003

2) Bauer G, Trinka E: Nonconvulsive status epilepticus and coma. Epilepsia 51: 177-190, 2010

3) Coutts SB, Hill MD, Hu WY, Sutherland GR: Hyperperfusion syndrome: Toward a stricter definition. Neurosurgery 53: 1053-1069, 2003

4) Fujimura M, Kaneta T, Shimizu H, Tominaga T: Symptomatic hyperperfusion after superficial temporal artery-middle cerebral artery anastomosis in a child with moyamoya disease. Childs Nerv Syst 23: 1195-1198, 2007

5) Fujimura M, Mugikura S, Kaneta T, Shimizu H, Tominaga T: Incidence and risk factors for symptomatic cerebral hyperperfusion after superficial temporal artery-middle cerebral artery anastomosis in patients with moyamoya disease. Surg 
Neurol 71: 442-448, 2009

6) Fukui K, Morioka T, Hashiguchi K, Kawamura T, Irita K, Hoka S, Sasaki T, Takahashi S: Relationship between regional cerebral blood flow and electrocorticographic activities under sevoflurane and isoflurane anesthesia. J Clin Neurophysiol 27: 110-115, 2010

7) Furuya K, Kawahara N, Morita A, Momose T, Aoki S, Kirino T: Focal hyperperfusion after superficial temporal arterymiddle cerebral artery anastomosis in a patient with moyamoya disease. J Neurosurg 100: 128-132, 2004

8) Hauf M, Slotboom J, Nirkko A, von Bredow F, Ozdoba C, Wiest R: Cortical regional hyperperfusion in nonconvulsive status epilepticus measured by dynamic brain perfusion CT. AJNR Am J Neuroradiol 30: 693-698, 2009

9) Hisada K, Morioka T, Nishio S, Muraishi M, Yamamoto T, Yoshida T, Fukui M: Magnetoencephalographic analysis of periodic lateralized epileptiform discharges (PLEDs). Clin Neurophysiol 111: 122-127, 2000

10) Kim JE, Oh CW, Kwon OK, Park SQ, Kim SE, Kim YK: Transient hyperperfusion after superficial temporal artery/middle cerebral artery bypass surgery as a possible cause of postoperative transient neurological deterioration. Cerebrovasc Dis 25: 580-586, 2008

11) Kohama M, Fujimura M, Mugikura S, Tominaga T: Temporal change of 3-T magnetic resonance imaging/angiography during symptomatic cerebral hyperperfusion following superficial temporal artery-middle cerebral artery anastomosis in a patient with adult-onset moyamoya disease. Neurosurg Rev 31: 451-455, 2008

12) Komoribayashi N, Ogasawara $K$, Kobayashi M, Saitoh H, Terasaka K, Inoue T, Ogawa A: Cerebral hyperperfusion after carotid endarterectomy is associated with preoperative hemodynamic impairment and intraoperative cerebral ischemia. J Cereb Blood Flow Metab 26: 878-884, 2006

13) Kuroda S, Kamiyama H, Abe H, Asaoka K, Mitsumori K: Temporary neurological deterioration caused by hyperperfusion after extracranial-intracranial bypass. Case report and study of hemodynamics. Neurol Med Chir (Tokyo) 34: 15-19, 1994

14) Lansberg MG, O’Brien MW, Norbash AM, Moseley ME, Morrell M, Albers GW: MRI abnormalities associated with partial status epilepticus. Neurology 52: 1021-1027, 1999
15) Medel R, Crowley RW, Dumont AS: Hyperperfusion syndrome following endovascular cerebral revascularization. Neurosurg Focus 26(3): E4, 2009 (review)

16) Meierkord H, Holtkamp M: Non-convulsive status epilepticus in adults: clinical forms and treatment. Lancet Neurol 6: 329-339, 2007

17) Naylor AR, Evans J, Thompson MM, London NJM, Abbott RJ, Cherryman G, Bell PRF: Seizures after carotid endarterectomy: Hyperperfusion, dysautoregulation or hypertensive encephalopathy. Eur J Vasc Endovasc Surg 26: 39-44, 2003

18) Ogasawara K, Inoue T, Kobayashi M, Endo H, Fukuda T, Ogawa A: Pretreatment with the free radical scavenger edaravone prevents cerebral hyperperfusion after carotid endarterectomy. Neurosurgery 55: 1060-1076, 2004

19) Reigel MM, Hollier LH, Sundt TM Jr, Piepgras DG, Sharbrough FW, Cherry KJ: Cerebral hyperperfusion syndrome: a cause of neurologic dysfunction after carotid endarterectomy. J Vasc Surg 5: 628-634, 1987

20) Shah AM, Vashi A, Jagoda A: Review article: Convulsive and non-convulsive status epilepticus: An emergency medicine perspective. Emerg Med Australas 21: 352-366, 2009

21) Szabo K, Poepel A, Pohlmann-Eden B, Hirsch J, Back T, Sedlaczek O, Hennerici M, Gass A: Diffusion-weighted and perfusion MRI demonstrates parenchymal changes in complex partial status epilepticus. Brain 128: 1369-1376, 2005

22) van Mook WNKA, Rennenberg RJMW, Schurinck GW, van Oostenbrugge RJ, Mess W, Hoffman PAM, de Leeuw PW: Cerebral hyperperfusion syndrome. Lancet Neurol 4: 877-888, 2005

23) Yoshimoto $T$, Houkin $K$, Kuroda S, Abe H, Kashiwaba T: Low cerebral blood flow and perfusion reserve induce hyperperfusion after surgical revascularization: case reports and analysis of cerebral hemodynamics. Surg Neurol 48: 132-138, 1997

Address reprint requests to: Takato Morioka, M.D., Ph.D., Department of Neurosurgery, Kyushu Rosai Hospital, 1-3-1 Kuzuharatakamatsu, Kokuraminami-ku, Kitakyushu, Fukuoka 800-0296, Japan. e-mail: takato@ns.med.kyushu-u.ac.jp 